\title{
Dual and Opposing Modulatory Effects of Serotonin on Crayfish Lateral Giant Escape Command Neurons
}

\author{
Terri Teshiba, ${ }^{1}$ Ashkan Shamsian, ${ }^{1}$ Bahram Yashar, ${ }^{1}$ Shih-Rung Yeh, ${ }^{2}$ Donald H. Edwards, ${ }^{3}$ and \\ Franklin B. Krasne ${ }^{1}$ \\ 1Department of Psychology, University of California, Los Angeles, Los Angeles, California 90095, 2Institute of Life \\ Sciences, National Tsing Hua University, 30013 Taiwan, Republic of China, and ${ }^{3}$ Department of Biology, Georgia State \\ University, Atlanta, Georgia 30302-4010
}

\begin{abstract}
Serotonin modulates afferent synaptic transmission to the lateral giant neurons of crayfish, which are command neurons for escape behavior. Low concentrations, or high concentrations reached gradually, are facilitatory, whereas high concentrations reached rapidly are inhibitory. The modulatory effects rapidly reverse after brief periods of application, whereas longer periods of application are followed by facilitation that persists for hours. These effects of serotonin can be reproduced by models
\end{abstract}

that involve multiple interacting intracellular signaling systems that are each stimulated by serotonin. The dependence of the neuromodulatory effect on dose, rate, and duration of modulator application may be relevant to understanding the effects of natural neuromodulation on behavior and cognition and to the design of drug therapies.

Key words: neuromodulation; crayfish; lateral giant; serotonin; facilitation; inhibition; escape behavior
Serotonin, octopamine, and GABA are all known to be present in crayfish and/or lobster nerve cord (Kravitz et al., 1976; Livingstone et al., 1980; Beltz and Kravitz, 1983; Glanzman and Krasne, 1986; Mulloney and Hall, 1990; Schneider et al., 1993) and can modulate the response of the crayfish lateral giant escape command neurons (LGs) to excitatory synaptic input. As a result, they regulate the excitability of escape (Glanzman and Krasne, 1983; Vu and Krasne, 1993; Yeh et al., 1996, 1997). As far as is known, the effect of GABA is always to inhibit and that of octopamine is to facilitate. However, the effect of 5-HT appears not to be fixed but to vary as a function of the animals' past social experience. When 5-HT is applied to the nerve cord of crayfish that have lived for several weeks as social subordinates, its effect is to inhibit EPSPs produced in the LGs by sensory activity, whereas 5-HT exposure to the cord of social dominants usually causes a facilitation of EPSP amplitude (Yeh et al., 1996, 1997). In social isolates, the picture is less clear, with different laboratories obtaining opposite effects (Glanzman and Krasne, 1983; Vu and Krasne, 1993; Yeh et al., 1996, 1997).

We report here that the LGs of social isolates have machinery for both serotonergic facilitation and inhibition and that the modulatory effect produced depends in complex ways on the dose and schedule of 5-HT exposure. These dependencies account for the opposing effects of serotonin obtained in different laboratories. We show that these complex dependencies could result from serotonergic activation of parallel and possibly interacting intracellular signaling cascades. However, whatever their mechanisms, variations in the qualitative form of aminergic neuromodulation

Received Oct. 26, 2000; revised April 5, 2001; accepted April 5, 2001.

This work was supported by National Institutes of Health Grants NS8108 (to F.B.K.) and NS26547 (to D.H.E.). We thank Ronald Harris-Warrick for helpful discussions and Paul Katz for suggestions on an early version of this manuscript.

Correspondence should be addressed to Frank Krasne, Department of Psychology, University of California, 1285 Franz Hall, Box 951963, Los Angeles, CA 90095-1563. E-mail: krasne@psych.ucla.edu.

Copyright (C) 2001 Society for Neuroscience $\quad 0270-6474 / 01 / 214523-07 \$ 15.00 / 0$ as a function of modulator dose, onset characteristics, and exposure duration may have important consequences for pharmacological therapies and ultimately be relevant to comprehending the ways in which neuromodulators are used naturally.

\section{MATERIALS AND METHODS}

Animals. Experiments were performed on either adult Procambarus clarkii measuring 8-9 $\mathrm{cm}$ rostrum to telson or on juveniles $2-3 \mathrm{~cm}$ in length. Crayfish were obtained from Atchalafaya Biological Supply (Raceland, LA) and kept isolated in $14 \times 20 \mathrm{~cm}$ polyethylene dishes for at least 2 weeks before experimentation. Adults and juveniles gave similar results, and their data were pooled. We avoided use of animals kept in the lab for many months because we believe that they have diminished responsiveness to applied 5-HT.

Experimental procedures. Animals were cooled gradually to $5^{\circ} \mathrm{C}$, the abdomen was pinned on Sylgard in crayfish saline, and the nerve cord was exposed dorsally as in Krasne (1969). Thereafter, experiments were at $\sim 20^{\circ} \mathrm{C}$. KCl-filled $(3 \mathrm{M}), \sim 10 \mathrm{M} \Omega$ recording electrodes were placed in proximal dendrites of LGs, usually in the sixth abdominal ganglion but in the third or fourth in a few experiments, and platinum wire stimulating electrodes were placed on roots $2-4$ (root 2 in third and fourth ganglion experiments). Test EPSPs were evoked every $2 \mathrm{~min}$ by a $0.2 \mathrm{msec}$ voltage pulse at stimulating electrodes. Exposure to 5-HT was begun when the amplitude of evoked EPSPs, which often drifted up or down after dissection and penetration, stabilized (usually $\sim 30 \mathrm{~min}$ ). Electrical activity was stored digitally for later analysis.

5-HT application. 5-HT was applied by superfusion in juveniles and by arterial perfusion (as in Mulloney et al., 1987) in adults. LOW concentration was $10^{-8} \mathrm{M}$ (perfusion) or $5 \times 10^{-6} \mathrm{M}$ (superfusion), and HIGH concentration was $5 \times 10^{-5} \mathrm{M}$ (perfusion) or $10^{-4} \mathrm{M}$ (superfusion). Exposure periods were either SHORT (10 min) or LONG (45 min or 40-60 min in initial experiments). For FAST applications, 5-HT was stepped to full concentration as rapidly as possible (within a few minutes). For SLOW applications, it was dripped so that full concentration was reached in $\sim 20-30$ min (assessed by replacing 5 -HT solutions with salt solutions and measuring conductance). 5-HT creatinine sulfate crystals (Research Biochemicals, Natick, MA) were stored with desiccant in a freezer (for not more than a few months) and dissolved just before use in 4 mM HEPES-buffered crayfish Ringer's solution, $\mathrm{pH}$ 7.2.

Data analysis. The EPSPs evoked by stimulation of ganglionic roots generally begin with an early depolarizing peak $(\alpha)$ caused by arrival of monosynaptic input and a slightly later peak $(\beta)$ produced by disynaptic input (Fig. $1 A, B$ ). Subsequent portions of the response reflect a mixture 
Figure 1. Effect of 5-HT on synaptic potentials evoked in LG by $0.2 \mathrm{msec}$ root shocks. $A$, Schematic of afferent portion of LG circuit. Chemical synapses (Chem excite) of sensory neurons on interneurons and rectifying electrical synapses (Rect elect) of sensory neurons and interneurons on LGs are indicated, as are the recording site at the LG axon initial segment and the soma of the unipolar LG. The monosynaptic innervation of LG causes an initial $(\alpha)$ depolarization, followed by another depolarization $(\beta)$ attributable to the disynaptic pathway; action potentials can be triggered by $\beta$ or sometimes $\alpha$ but not later parts of the EPSP, which reflects a complex mix of excitation and depolarizing inhibition. $B$, Time plots of normalized $\alpha$ (open circles) and $\beta$ ( filled circles) component amplitudes for illustrative experiments under each of the indicated conditions of 5-HT delivery. 5-HT exposure as indicated by solid bars, with the width of the bar approximately indicating 5-HT concentration. Test stimuli were at 2 min intervals. To the right of each graph are sample synaptic potentials recorded before 5-HT application (thin continuous lines), at the end of 5-HT application (solid bold lines), and after $30 \mathrm{~min}$ of 5-HT washout (dashed bold lines).
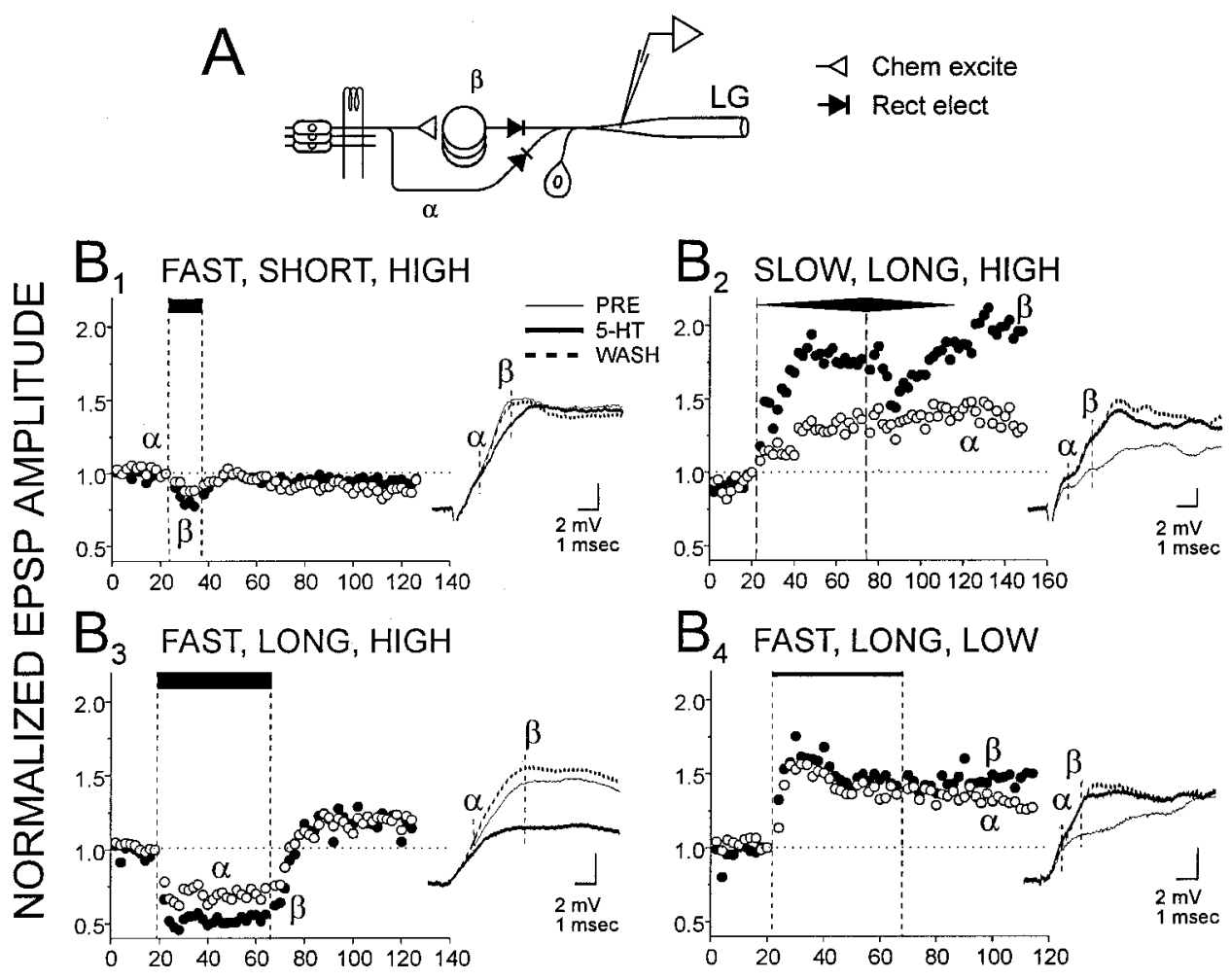

TIME (min)

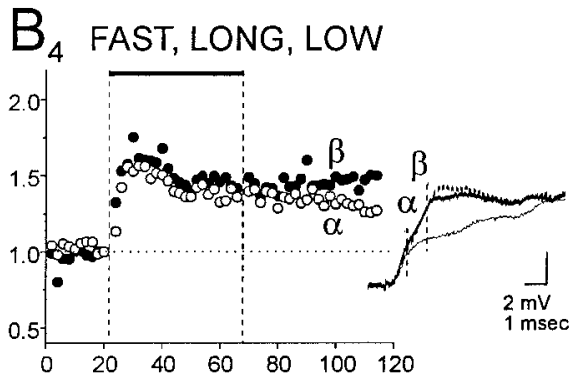

TIME (min) of polysynaptic excitatory and inhibitory inputs that follow the stimulus. In analyzing our data, we measured membrane potential at the times at which $\alpha$ and $\beta$ peaks were located at the beginning of the experiment, even when these peaks subsequently shifted somewhat. In some cases, the $\beta$ peak was not unimodal. We then usually measured the amplitude of the earlier peak. For graphing, EPSP amplitudes were normalized to their value on the last control EPSP before introduction of 5-HT. Although we attempted to set initial EPSP levels so that LG spikes, which interfered with measurement of EPSP amplitudes, would be unlikely, sometimes serotonin increased EPSPs enough to cause spikes. In these cases, we entered into analyses an EPSP amplitude $20 \%$ higher than the largest EPSP amplitude of the experiment that was not contaminated by a spike. Mean EPSP amplitudes were calculated for the last 5 pre-serotonin trials, the last 5 serotonin trials (the last three in the case of the SHORT condition), and wash trials 15-19 (30 min after the start of serotonin washout). Statistical analyses were on differences between the mean EPSP amplitude at the time point of interest and the pre-serotonin mean. Statistical tests were two-tailed $t$ tests.

\section{RESULTS}

The LG receives converging synaptic input from primary mechanosensory afferents and mechanosensory interneurons (Fig. 1A) that produce monosynaptic $(\alpha)$ and disynaptic $(\beta)$ EPSPs, respectively (Fig. 1B) (Krasne, 1969); LG spikes and reflexive tail flips are usually produced only when the $\beta$ peak exceeds firing level (Krasne, 1969; Olson and Krasne, 1981), although under some circumstances the $\alpha$ component can also become large enough to trigger spikes and behavior (Edwards et al., 1994).

The effect of rapid application of 5-HT (maximum level reached within 1-2 $\mathrm{min}$ ) for $10 \mathrm{~min}$ or less at a high concentration (the FAST-SHORT-HIGH condition; see Materials and Methods) is shown in Figure $1 B_{1}$ for an illustrative preparation. Both the $\alpha$ and $\beta$ components of the LG EPSP declined during the 5 -HT but were restored to their baseline values after several minutes of 5-HT washout. This same pattern can be seen to hold for the averaged normalized $\beta$ component in Figure 2, which plots the time course of 5-HT effects for all conditions, superimposed to facilitate comparison. Figure $3 A$ shows mean EPSP amplitudes at the end of 5-HT exposure and after $30 \mathrm{~min}$ of washout. The decline of the $\beta$ component is statistically reliable at the end of the 5-HT exposure $(p<0.05)$ but not significantly different from baseline after $30 \mathrm{~min}$ of wash. As in several conditions of this study, the effects of 5-HT on the $\alpha$ component paralleled those on the $\beta$ component but were not statistically reliable. However, in

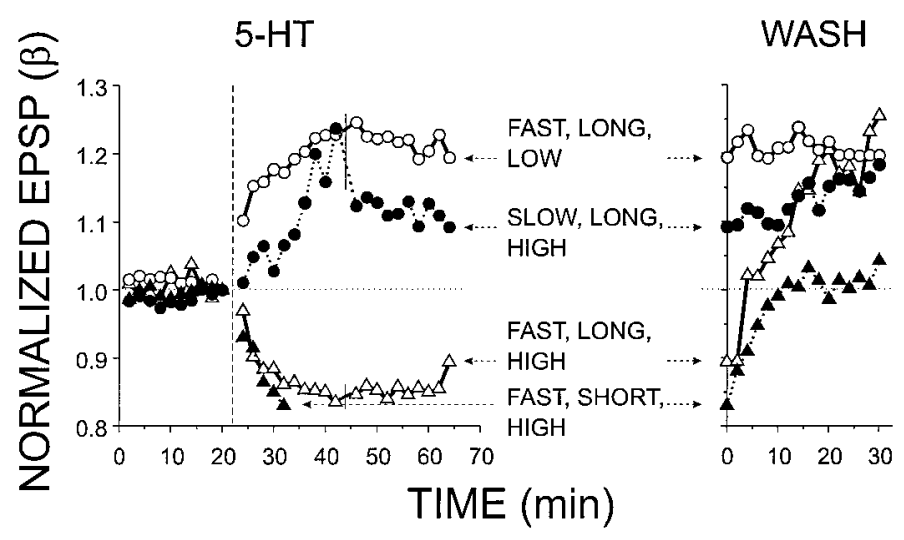

Figure 2. Group mean time courses of $\beta$ component EPSP amplitude during and after 5-HT exposure. $\beta$ component amplitudes were normalized by their values on the last stimulation before the start of 5-HT exposure. Exposure began at the dashed vertical line. The short vertical line segments at $\sim 45$ min indicate omission of a few time points for those preparations that were exposed to 5-HT for $>40 \mathrm{~min}$. The EPSP amplitude on the last trial in 5-HT is taken as the 0 time point for the washout graphs. 


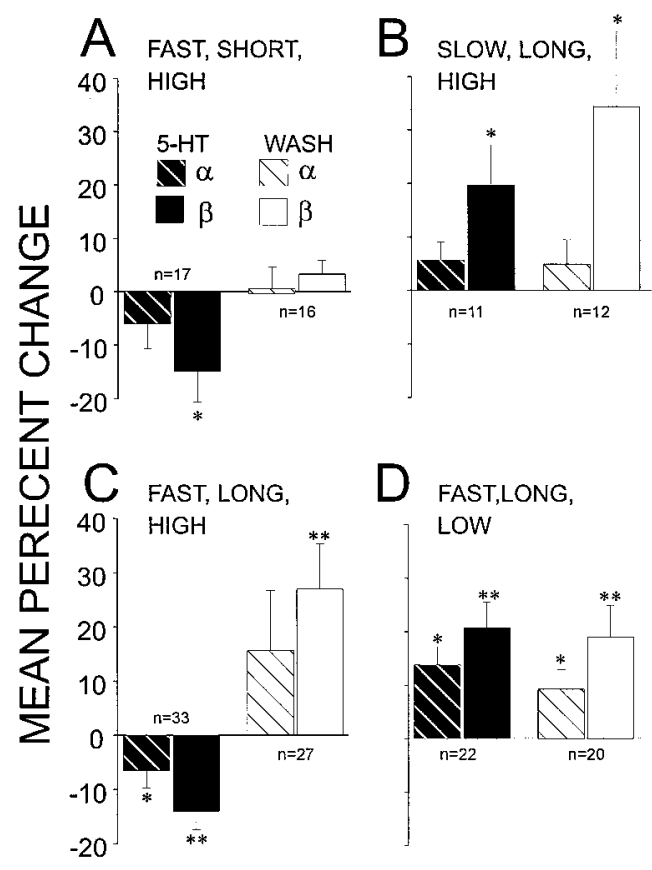

Figure 3. Group means and SEs of the percentage change in EPSP amplitudes at the end of 5-HT exposure and after $30 \mathrm{~min}$ of wash for each regimen of 5-HT application. Significance levels are indicated by asterisks: ${ }^{*} p<0.05 ; * * p<0.01$. Numbers of cases in each condition $(n)$ are shown near the $x$-axis.

two previous studies in which 5-HT exposures comparable with those of the FAST-SHORT-HIGH conditions were examined (Glanzman and Krasne, 1983; Vu and Krasne 1993), the decline of the $\alpha$ component amplitude, although generally smaller than that of the $\beta$ component, was reliable. Moreover, if the data from the FAST-SHORT-HIGH condition are combined with the identically obtained data from the first $10 \mathrm{~min}$ of 5-HT exposure in the FAST-LONG-HIGH condition, the mean percentage decline of the $\alpha$ component at the end of $10 \mathrm{~min} 5$-HT exposure does become statistically significant $(p<0.05)$.

Very different results were obtained when the same concentration of serotonin was applied much more slowly, so that the modulator reached its maximum level only after 20-30 min (SLOW-LONG-HIGH condition). Under this condition, facilitation rather than inhibition of both LG EPSP components developed, and the facilitation became persistent, long outlasting removal of the serotonin (Figs. $1 B_{2}, 2,3$ ). In both Figures 1 and 2 , the $\beta$ component amplitude appears to fall during the latter half of the period of 5-HT exposure and to rise again during wash. This was seen in half of the preparations, but it was not statistically reliable across the full population. The $\alpha$ component changes, which were in the same direction as the statistically reliable $\beta$ component changes, were not statistically reliable (Fig. $3 B$ ). However, because increases in the $\alpha$ component in comparable previous work (Yeh et al., 1996, 1997) were statistically reliable, we believe that the increases, although insignificant here, are real.

These findings for the FAST-SHORT-HIGH and SLOWLONG-HIGH conditions duplicate the opposing results of previous studies (Glanzman and Krasne, 1983; Vu and Krasne, 1993; Yeh et al., 1996, 1997) and define the circumstances under which they are obtained.
With slow 5-HT application, EPSP facilitation began when the gradually rising concentration of 5-HT was still quite low (consistent with Yeh et al., 1997). This suggested the possibility that the concentration threshold of facilitation was lower than that needed to cause EPSP reduction. We thus examined the effects of rapid application of a low concentration of 5-HT that was maintained for 45-60 min (FAST-LONG-LOW condition; see Materials and Methods). This procedure produced prompt facilitation of the LG EPSP instead of inhibition, and the facilitation persisted after washout of 5-HT, as in the response to the SLOW-LONG-HIGH application (Figs. $1 B_{4}, 2,3$ ). The increases of both the $\alpha$ and $\beta$ components at the end of 5-HT application and after $30 \mathrm{~min}$ of wash were statistically significant (Fig. 3D).

Because persistent facilitation was seen during washout in both the SLOW-LONG-HIGH and the FAST-LONG-LOW conditions, but not in the FAST-SHORT-HIGH condition, we examined the effect of FAST-LONG-HIGH exposures. Consistent with the findings for the FAST-SHORT-HIGH condition, the initial effect of the 5-HT was inhibitory. This inhibition persisted throughout the $\sim 45$ min period of 5 -HT exposure (Figs. $1 B_{3}, 2$, $3 C$; both $\alpha$ and $\beta$ component reductions statistically significant). However, when the 5-HT was washed out, the inhibition gave way to a persistent facilitation (Figs. $1 B_{3}, 2$ ). This facilitation was statistically reliable for the $\beta$ but not the $\alpha$ component (Fig. 3C). Thus, persistent facilitation occurred in the aftermath of all LONG exposures, regardless of the initial effect of serotonin (Figs. $1 B_{2}-B_{4} ; 2, W A S H$ curves; $3 B-D$, open bars). In many of the present experiments, facilitation persisted for $>1 \mathrm{hr}$ without decline, and in a previous report using slow application (Yeh et al., 1997), facilitation of both $\alpha$ and $\beta$ were routinely seen to persist throughout $1 \mathrm{hr}$ of wash and in some cases seen to persist unabated during $5 \mathrm{hr}$ of wash.

The LGs became depolarized during exposure to 5-HT in all conditions and all experiments and by approximately the same amount in each condition (mean $\pm \mathrm{SD}, 2.7 \pm 1.3$ ). Depolarization also occurred during facilitation in Yeh et al. (1997) and during inhibition produced by exposures, comparable with our FAST, HIGH conditions (Vu and Krasne, 1993).

\section{DISCUSSION}

These experiments have uncovered two distinct forms of serotonergic modulation affecting the LGs in socially isolated crayfish, a facilitation that is produced by low concentrations of 5-HT ("lowthreshold facilitation") and an inhibition that is produced by high 5-HT concentrations ("high-threshold inhibition"), both of which are associated with depolarization of the LGs.

The fact that 5-HT effects were generally more pronounced for the $\beta$ component than for the monosynaptic $\alpha$ component might indicate that the effects of 5-HT on the $\beta$ component are in part attributable to modulation of the first-order synapse of the disynaptic pathway. However, inhibition of the $\alpha$ component was statistically significant at both 10 and 45-60 min after rapid introduction of a high concentration of 5-HT, and facilitation of the monosynaptic response was statistically reliable during exposure to low concentrations of 5-HT. Reliable facilitation and inhibition of monosynaptic $(\alpha)$ input to the LGs have also been seen in previous studies (Glanzman and Krasne, 1983; Vu and Krasne, 1993; Yeh et al., 1996, 1997). Additionally, the fact that 5-HT altered the membrane potential of the LGs in both this and previous studies and that LG input conductance increased in 
association with the inhibition ( $\mathrm{Vu}$ and Krasne, 1993) and decreased in association with the facilitation (Yeh et al., 1997) supports the view that both facilitatory and inhibitory effects of 5-HT in isolates are attributable at least in part to modulation directly at the level of the LG and/or synapses directly on it.

The same SLOW-LONG-HIGH application of serotonin that here and in previous studies (Yeh et al., 1996, 1997) produced facilitation in social isolates produces inhibition of the response of the LG in social subordinates (Yeh et al., 1997). Because the inhibition studied in subordinates, unlike the high-threshold inhibition studied here, is associated with LG hyperpolarization, it appears to be a distinct type of serotonergic modulation of LG, different from either of those observed here.

The present experiments also add to the complexity of serotonergic modulation of LG in that directly opposite effects of serotonin are obtained not only in animals with different social histories but also in animals of a given history when different patterns of serotonin application are used. It remains to be determined whether the effect of serotonin on the LGs in subordinates is also sensitive to the pattern of application.

\section{Possible mechanisms}

The cellular mechanisms that cause the modulatory effects of serotonin to depend on the concentration, application rate, and duration of exposure are unknown, but some preliminary conclusions may be drawn. The results shown here suggest that 5-HT exposure can activate two parallel intracellular signaling pathways, a lower-threshold facilitatory pathway, and a higherthreshold inhibitory pathway. These pathways might be activated by either different 5-HT receptors or different levels of a common initial second messenger (as in Artola and Singer, 1993; Lisman, 1994; Bear, 1995).

The production of inhibition when higher concentrations of 5-HT are introduced rapidly but facilitation when the same concentrations are reached slowly suggests that intracellular signals generating one form of modulation may interfere with early steps involved in producing the other, as shown in Figure $4 A_{1}$. If such mutual suppression operated, then whichever modulatory pathway became active first would prevent development of the other form of modulation and, thus, would dominate. With gradual application of 5-HT, the lower-threshold facilitatory pathway would dominate because it would be engaged first. On the other hand, if the high-threshold inhibitory pathway had a faster response time than the facilitatory signaling pathway, then sudden increases of 5-HT concentration to high levels would cause inhibition rather than facilitation. This is by no means the only possible explanation of the application rate sensitivity we observed, but it was the most plausible one we could think of.

Serotonergic facilitation that lasts for hours after prolonged but not after brief 5-HT exposures is reminiscent of a similar effect at sensorimotor synapses of Aplysia. There, hours long facilitation can result from either translation-dependent (but transcriptionindependent) processes (Ghirardi et al., 1995; Mauelshagen et al., 1996, 1998) or entirely post-translational processes (Ghirardi et al., 1995). The exact mechanisms operating either in Aplysia or here are unknown, and there are many possibilities. For example, such properties can in principle arise from mutual facilitation between signaling pathways, as illustrated by modeling of the interactive properties of mitogen-activated protein (MAP) kinase and protein kinase C (Bhalla and Iyengar, 1999). Another possibility is that the process that normally destroys facilitatory precursors gradually inactivates and allows the
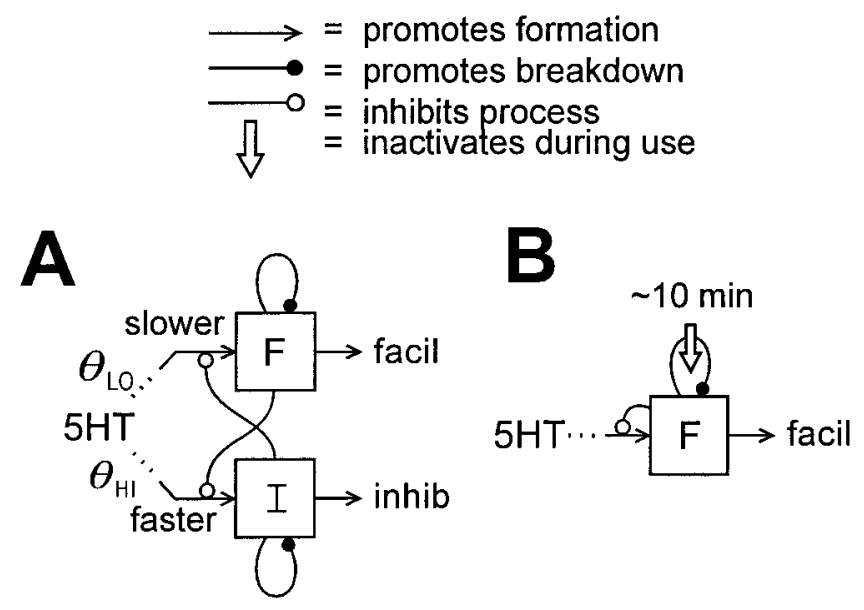

Figure 4. Illustrative explanations of key data features. Here and in Figure 5, boxes indicate activated receptors, activated G-proteins, second messengers, or activated enzymes such as kinases. Lines with arrowheads indicate stimulation of production of the targeted factor, possibly via a number of unspecified steps. Filled circles indicate processes that cause breakdown or inactivation of a factor. Open circles indicate inhibition of a process. Open arrows indicate usage-produced decline in the efficacy of a process. $A$, Possible mechanism for dependence on rate of 5-HT application (see Discussion). $\theta_{L O}$, Low-threshold facilitatory pathway; $\theta_{H I}$, highthreshold inhibitory pathway (see Discussion). $B$, Possible mechanism for 5-HT duration-dependent persistence of facilitation (see Discussion).

remaining precursors and resultant facilitation to persist, as shown in Figure $4 B$. The mechanisms by which G-proteincoupled receptors adapt (Ferguson and Caron, 1998; Lefkowitz, 1998) provides a possible mechanism, although at a shorter time scale, for such inactivation.

\section{Ability of mechanisms to account for findings}

To show that signaling pathway interactions really could cause the phenomena we observed, we constructed a specific computational model (Fig. 5A) that combined the mutual suppression and the inactivation processes just discussed; the quantitative details of the model are given in Appendix. The model consists of interacting facilitatory $(F)$ and inhibitory $(I)$ pathways activated by serotonin $(S)$. Each pathway has three reaction steps in which an initial product $\left(F_{\mathrm{o}}\right.$ or $I_{\mathrm{o}}$ ) increases as a sigmoidal function of $\log$ $S$ and is converted to secondary and tertiary products $\left(F_{1}, F_{2}\right.$, or $\left.I_{1}, I_{2}\right)$. Products increase at a rate proportional to the amount of precursor (Fig. $5 A$, processes $3,7,10,11$ ) and are broken down at a rate proportional to their own amount (Fig. $5 A$, processes 5,9 , $13,14)$, and where necessary, feedback inhibition was used to avoid run-away growth of products (Fig. $5 A$, processes 4,8 ). Whichever form of modulation becomes established first, be it facilitation or inhibition, dominates because product $F_{2}$ suppresses the production of $I_{2}$ and conversely (Fig. $5 A$, process 12; compare with Fig. $4 A$ ). Facilitation persists after extended serotonin exposure because breakdown of $F_{1}$ (Fig. 5A, process 5) inactivates (Fig. $5 A$, process 6 ; compare with Fig. $4 B$ ). The final products $\left(F_{2}\right.$ and $\left.I_{2}\right)$ determine multiplicative factors that adjust EPSP amplitude; the facilitatory multiplier was constructed to increase the EPSP by a percentage proportional to the amount of facilitatory product and correspondingly for the inhibitory multiplier and the final inhibitory product. EPSP amplitudes predicted by the model under the four different conditions of 5-HT application are shown in Figure $5 B$.

The FAST-SHORT-HIGH 5-HT stimulus (Fig. $5 B_{1}$ ) is inhib- 


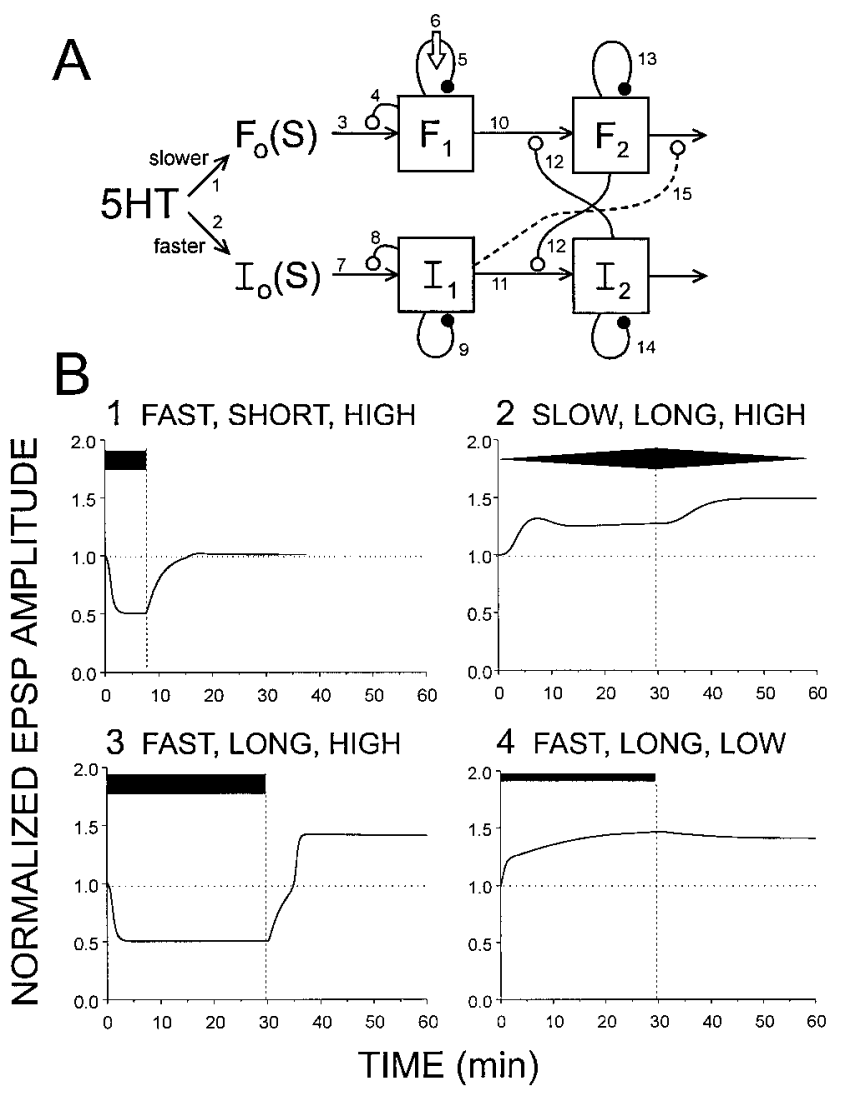

Figure 5. A model that predicts the observed results. $A$, The model; Discussion contains a qualitative explanation, and Appendix contains full quantitative characterization. Symbols are defined in the legend of Figure 4. $F_{0}-F_{2}$ and $I_{1}-I_{2}$ are key intermediaries in the conjectured facilitatory and inhibitory pathways, respectively; processes are numbered for reference and correspond to equation numbers in Appendix. $B_{1}-B_{4}$, EPSP amplitudes predicted by the model under the four different conditions of 5-HT application. The width of the dark bars approximately indicate concentration of 5-HT.

itory because the early, rapid response of the inhibitory pathway suppresses production of $F_{2}$. Facilitation is not activated long enough for process 5 (Fig. $5 A$ ) to inactivate; therefore, $F_{1}$ does not become self-sustaining and consequently facilitation fails to develop during wash.

The gradual increase in 5-HT concentration during the SLOW-LONG-HIGH condition (Fig. $5 B_{2}$ ) promotes facilitation because the low-threshold facilitatory response $\left(F_{2}\right)$ develops first and $F_{2}$ laterally inhibits the production of $I_{2} . F_{1}$ becomes selfsustaining because its breakdown inactivates; thus, facilitation persists during wash. The trend in the biological data for facilitation to diminish after extended exposure to 5-HT and to rise further during wash was generated by introducing feedforward inhibition of the effects of $F_{2}$ by $I_{1}$ (Fig. $5 A$, process 15 ).

The FAST-LONG-HIGH stimulus (Fig. $5 B_{3}$ ) causes inhibition for the same reasons as does the FAST-SHORT-HIGH stimulus. $F_{1}$ becomes self-sustaining because $F_{1}$ is elevated long enough for the breakdown reaction (Fig. $5 A$, process 5 ) to inactivate. Therefore, $F_{2}$ is produced once inhibition of its production by $I_{2}$ (Fig. $5 A$, process 12) ceases, with the consequence that facilitation becomes manifest during washout of 5-HT.

The FAST-LONG-LOW stimulus (Fig. 5B $_{4}$ activates the lower-threshold facilitatory pathway for long enough to result in self-sustaining elevation of $F_{1}$, but it fails to activate the higher- threshold inhibitory pathway. Thus, persistent facilitation is produced.

The signaling pathways mediating the effects of serotonin on the LG are not yet known. Thus, attempts to suggest specific signaling pathways and interactions that could implement this model, which is itself only one of a number that could reproduce our findings, would be premature. However, it should be pointed out that the model is not unrealistic in terms of types of mechanisms that are known to operate in cellular signaling pathways. Thus, $F_{1}$ (or $I_{1}$ ) in the model might plausibly correspond to a second messenger, such as cAMP or diacylglycerol, and $F_{2}\left(\right.$ or $\left.I_{2}\right)$ to the activated form of the associated protein kinase of the second messenger. The activated kinases might then alter membrane conductances by directly phosphorylating channel proteins, the decreased conductance and depolarization associated with low-threshold facilitation being consistent with closure of a $\mathrm{K}^{+}$ channel and the increased conductance and depolarization associated with high-threshold inhibition being consistent with opening of a $\mathrm{Cl}^{+}$channel. The suppression of $F_{2}$ production by $I_{2}$ would then result if the activated kinase corresponding to $I_{2}$ were to inhibit the activation of the kinase corresponding to $F_{2}$ by phosphorylating suitable sites on its regulatory region and conversely (as in inhibition of MAP kinase and CaM kinase kinase activation by PKA) (D'Angelo et al., 1997; Wayman et al., 1997; Soderling, 1999). The $F_{1}$ persistence postulated in the model could be attributable to inactivation of an enzymatic reaction breaking down the second messenger (see above) or to some other mechanism leading to prolonged elevation of secondmessenger levels such as those responsible for prolonged elevation of DAG in some systems (Nishizuka, 1995). Given this set of proposals, feedforward inhibition (Fig. $5 A$, process 15 ) would have to be explained by postulating that the second messenger corresponding to $I_{1}$ causes a reduction in the extent to which the phosphorylation caused by facilitatory kinase closes the $\mathrm{K}^{+}$channel. Because some second messengers can directly gate ion channels (Yau, 1994), this effect might be direct.

Alternatively, $F_{1}$ and $I_{1}$ could correspond to two different activated protein kinases and $F_{2}$ and $I_{2}$ to the sets of phosphorylated proteins that are the targets of the kinases. Then, if the activated kinase corresponding to $I_{1}$ were to inhibit the catalytic action of the activated kinase corresponding to $F_{2}$ by phosphorylating it, and conversely, the mutual inhibition postulated by the model would be accomplished. The persistence of $F_{1}$ would here be equivalent to the sustained activation of the kinase to which it corresponds; sustained activation of PKA in fact occurs in Aplysia after suitably extended periods of serotonin application (Muller and Carew, 1998). Under this set of assumptions, feedforward inhibition would have to be explained by assuming that the activated kinase corresponding to $I_{1}$ reduces the extent to which the phosphorylation of the $\mathrm{K}^{+}$channel catalyzed by the facilitatory kinase effectively closes the $\mathrm{K}^{+}$channel; the $I_{1}$ kinase could do this by directly phosphorylating the $\mathrm{K}^{+}$channel at a suitable regulatory site.

More specific and more complete hypotheses must await identification of the signaling pathways recruited in the LGs by 5-HT. However, whatever the actual mechanisms, our simulations show that the dependence of the effect of 5-HT on dose, rate, and duration of 5-HT application may in principle be understood in terms of complex interactions between second messengers and enzymes in intracellular signaling systems. Interactions of this kind provide a capability for logical operations, memory, and 
temporal pattern generation based on intracellular biochemical networks rather than on multicellular neural networks (Katz, 1999; Weng et al., 1999).

\section{Functional importance}

These different application regimens may mimic the different ways that naturally released 5-HT can reach the LG neuron. A serotonergic neuron has been found that projects caudally along the dorsal aspect of the LGs and has terminal varicosities in apparent contact (at the light microscopic level) with the initial dendritic segment of the LG in each abdominal ganglion (Yeh et al., 1997). Activation of this serotonergic neuron could cause the concentration of serotonin in the vicinity of LG to rise quickly to high levels, mimicking the FAST-HIGH application regimen. Serotonin is also a hormone in the blood of crayfish and lobsters (Beltz and Kravitz, 1983); the lack of a blood-brain barrier allows serotonin in the blood to reach the LG, but it presumably does so more slowly and at lower concentrations than with synaptic release, perhaps mimicking the SLOW and LOW application regimens of this study.

The functional significance of serotonergic modulation of LG excitation is not yet clear. However, because injected serotonin seems to have effects on social behavior (Huber et al., 1997) and because its effects on the LGs are social experience dependent, it seems likely that the modulation is important during agonistic interactions. There also is evidence for both increases in medial giant-mediated escape (Herberholz et al., 2001) and decreases of lateral giant-mediated escape (Krasne et al., 1997) during social interactions of freely behaving crayfish, although a role for serotonin in such modulation has not been established.

The factors determining 5-HT exposure pattern in crayfish are not unique. Cortical targets located close to the release sites of serotonergic neurons projecting from the raphe nuclei might be expected to experience potentially large, rapidly changing levels of 5-HT exposure, whereas more distant targets would experience smaller, slower changes (Stamford et al., 2000). If similar to crayfish, these proximal and distal targets might be modulated very differently. Understanding how intracellular signaling systems are tailored to respond to different aspects of the 5-HT signal may guide both the study of the cognitive and behavioral responses to natural neuromodulation and the design of drug therapies.

\section{APPENDIX}

This appendix provides a mathematical characterization of the model described in Discussion and diagramed in Figure $5 A$. Variable names correspond to those of the figure, and equation numbers correspond to the numbered processes of the figure.

Serotonin at concentration $S$ produces initial intracellular facilitatory and inhibitory factors, $F_{0}$ and $I_{0}$, that are sigmoidal functions of $\log S$. Specifically,

$$
F_{0}=1 /\left(1+e^{-A\left(\log S-\log S_{0}\right)}\right)-z_{0},
$$

where $A=2, \log S_{0}=-8$, and $z_{0}$ is chosen so that $F_{0}=0$ when $\log S=-10$,

and

$$
I_{0}=1 /\left(1+e^{-A\left(\log S-\log S_{0}\right)}\right)-z_{0},
$$

where $A=2, \log S_{0}=-5$, and $z_{0}$ is chosen so that $I_{0}=0$ when $\log S=-10$.

$F_{1}$ is produced at a rate proportional to $F_{0}$ but is attenuated by a factor, $r_{\mathrm{epi} F}$, because of endpoint inhibition, which is constructed to keep $F_{1}$ from exceeding unity even when breakdown of $F_{1}$ is totally inactivated (see below). Thus,

$$
\text { rate of } F_{1} \text { production }=\alpha_{F_{1}} \cdot r_{\mathrm{epi} F}\left(F_{1}\right) \cdot F_{0}(S)
$$

where

$$
r_{\mathrm{epi} F}\left(F_{1}\right)=1-F_{1} .
$$

$F_{1}$ breaks down at a rate proportional to its concentration, but the rate of degradation is attenuated by an inactivation factor, $r_{\mathrm{i}}$, that declines as breakdown occurs. This inactivation of $F_{1}$ breakdown is responsible for the persistence of $F_{1}$ when 5 -HT is removed after long exposures. Thus,

$$
\text { rate of } F_{1} \text { breakdown }=\beta_{F_{1}} \cdot r_{\mathrm{i}}(t) \cdot F_{1},
$$

where

$$
\Delta r_{\mathrm{i}} / \Delta t=-\gamma_{r_{\mathrm{i}}} r_{\mathrm{i}}(t) \text { when } F_{1}>0 ; \quad r_{\mathrm{i}}(0)=1 .
$$

Taking into account both production and breakdown,

$$
\Delta F_{1} / \Delta t=\alpha_{F_{1}} r_{\mathrm{epi} F}\left(F_{1}\right) F_{0}(S)-\beta_{F_{1}} r_{\mathrm{i}}(t) F_{1} .
$$

Production and breakdown of $I_{1}$ are computed similarly exceptthat breakdown of $I_{1}$ does not inactivate. Thus,

$$
\text { rate of } I_{1} \text { production }=\alpha_{I_{1}} \cdot r_{\text {epi } I}\left(I_{1}\right) \cdot I_{0}(S),
$$

where endpoint inhibition is given by

$$
r_{\text {epir }}\left(I_{1}\right)=\left(1+\beta_{I_{1}} / \alpha_{I_{1}}\right)-I_{1},
$$

which prevents $I_{1}$ from exceeding unity, and

$$
\text { rate of } I_{1} \text { breakdown }=\beta_{I_{1}} I_{1} \text {. }
$$

Taking into account both production and breakdown,

$$
\Delta I_{1} / \Delta t=\alpha_{I_{1}} r_{\text {epir }}\left(I_{1}\right) I_{0}(S)-\beta_{I_{1}} I_{1} .
$$

$F_{2}$ and $I_{2}$ are each produced at rates proportional to their precursors, but production of each intermediary is inhibited by the other (mutual inhibition, $r_{\mathrm{mi}}$ ). Thus,

$$
\text { rate of } F_{2} \text { production }=\alpha_{2} \cdot r_{\text {mi }}\left(I_{2}\right) \cdot F_{1}
$$

and

$$
\text { rate of } I_{2} \text { production }=\alpha_{2} \cdot r_{\mathrm{mi}}\left(F_{2}\right) \cdot I_{1} \text {, }
$$

where the mutual inhibition attenuation factor is given by

$$
r_{\text {mi }}(x)=1 /\left(1+\gamma_{\text {mi }} x^{2}\right),
$$

with the argument, $x$, being either $I_{2}$ or $F_{2}$.

As above, breakdown of $F_{2}$ and $I_{2}$ are given by

$$
\begin{aligned}
& \text { rate of } F_{2} \text { breakdown }=\alpha_{2} \cdot F_{2}, \\
& \text { rate of } I_{2} \text { breakdown }=\alpha_{2} \cdot I_{2} .
\end{aligned}
$$

Hence,

$$
\begin{gathered}
\Delta F_{2} / \Delta t=\alpha_{2}\left[r_{\mathrm{mi}}\left(I_{2}\right) F_{1}-F_{2}\right], \\
\Delta I_{2} / \Delta t=\alpha_{2}\left[r_{\mathrm{mi}}\left(F_{2}\right) I_{1}-I_{2}\right] .
\end{gathered}
$$


Finally, EPSP amplitudes are assumed to be changed by factors proportional to final facilitatory and inhibitory products. Thus,

$$
\mathrm{EPSP}_{\text {normalized }}=\left[1+\sigma_{F} F_{2}\right]\left[1-\sigma_{I} I_{2}\right] .
$$

However, in approximately half of our experiments under the SLOW-LONG-HIGH condition and in the mean curves of Figure 2, the elevated $\beta$ component of the EPSP fell somewhat as 5-HT concentration increased and then grew again as 5-HT was washed out. Although this effect was not statistically reliable, it was sufficiently common (and clear in some individual experiments) that we wanted our model to be able to emulate it. It could not be explained by production of small amounts of $I_{2}$ as the slowly rising 5-HT concentration grew, because mutual inhibition necessarily drives $I_{2}$ to zero when 5-HT concentration rises slowly. Therefore, to account for this feature of the data, we assumed that $I_{1}$ attenuates $F_{2}$-caused facilitation by a factor, $r_{\mathrm{ffi}}$ (feedforward inhibition) via the dashed pathway of Figure 5. Thus,

$$
\mathrm{EPSP}_{\text {normalized }}=\left[1+\sigma_{F} r_{f f i} F_{2}\right]\left[1-\sigma_{I} I_{2}\right]
$$

where

$$
r_{f f i}\left(I_{1}\right)=1 /\left(1+\gamma_{f f i} I_{1}\right) .
$$

Simulations were done with this feedforward inhibition operative. When it is omitted, the prewash dip and postwash rise of EPSP amplitude seen in Figure $5 B_{2}$ do not occur.

For computations, 4000 updates were taken to correspond to 30 min of real time or $0.45 \mathrm{sec}$ per update. For HIGH conditions, log $S$ was set to -3 , and for LOW to -7 . FAST applications were simulated by steps of $\log S$ from -10 to final concentration, whereas for SLOW application $\log S$ was started at -10 and altered over time according to $\Delta \log S / \Delta t=0.008\left(\log S_{\text {final }}-\log \right.$ $S$ ). LONG was equal to 4000 iterations and SHORT to 1000 , corresponding nominally to 30 and 7.5 min of 5-HT application, respectively. Parameters used in simulations were as follows:

$$
\begin{array}{r}
\alpha_{F_{1}}=0.01 ; \quad \beta_{F_{1}}=0.01 ; \quad \alpha_{I_{1}}=0.008 ; \quad \beta_{I_{1}}=0.003 ; \\
\alpha_{2}=0.025 ; \quad \sigma_{F}=\sigma_{I}=0.5 ; \quad \gamma_{\mathrm{ri}}=0.001 ; \\
\gamma_{\mathrm{mi}}=1250 ; \quad \text { and } \gamma_{f i}=0.8 .
\end{array}
$$

\section{REFERENCES}

Artola A, Singer W (1993) Long-term depression of excitatory synaptic transmission and its relationship to long-term potentiation. Trends Neurosci 16:480-487.

Bear MF (1995) Mechanism for a sliding synaptic modification threshold. Neuron 15:1-4.

Beltz BS, Kravitz EA (1983) Mapping of serotonin-like immunoreactivity in the lobster nervous system. J Neurosci 3:585-602.

Bhalla US, Iyengar R (1999) Emergent properties of networks of biolgical signaling pathways. Science 283:381-387.

D'Angelo G, Lee H, Weiner RI (1997) cAMP-dependent protein kinase inhibits the mitogenic action of vascular endothelial growth factor and fibroblast growth factor in capillary endothelial cells by blocking Raf activation. J Cell Biochem 67:353-366.

Edwards DH, Fricke RA, Barnett LD, Yeh SR, Leise EM (1994) The onset of response habituation during the growth of the lateral giant neuron of crayfish. J Neurophysiol 72:890-898.

Ferguson SS, Caron MG (1998) G protein-coupled receptor adaptation mechanisms. Semin Cell Dev Biol 9:119-127.

Ghirardi M, Montarolo PG, Kandel ER (1995) A novel intermediate stage in the transition between short- and long-term facilitation in the sensory to motor neuron synapse of Aplysia. Neuron 14:413-420.

Glanzman DL, Krasne FB (1983) Serotonin and octopamine have opposite modulatory effects on the crayfish's lateral giant escape reaction. J Neurosci 3:2263-2269.

Glanzman DL, Krasne FB (1986) 5,7-Dihydroxytryptamine lesions of crayfish serotonin-containing neurons: effect on the lateral giant escape reaction. J Neurosci 6:1560-1569.

Herberholz J, Issa FA, Edwards DH (2001) Patterns of neural circuit activation and behavior during dominance hierarchy formation in freely behaving crayfish. J Neurosci 21:2759-2767.

Huber R, Smith K, Delago A, Isaksson K, Kravitz EA (1997) Serotonin and aggressive motivation in crustaceans: altering the decision to retreat. Proc Natl Acad Sci USA 94:5939-5942.

Katz PS (1999) In: Beyond neurotransmission: neuromodulation and its importance for information processing (Katz PS, ed), pp 1-28. Oxford: Oxford UP.

Krasne FB (1969) Excitation and habituation of the crayfish escape reflex: the depolarizing response in lateral giant fibres of the isolated abdomen. J Exp Biol 50:29-46.

Krasne FB, Shamsian A, Kulkarni R (1997) Altered excitability of the crayfish lateral giant escape reflex during agonistic encounters. J Neurosci 17:709-716.

Kravitz EA, Evans PD, Talamo BR, Wallace BG, Battelle BA (1976) Octopamine neurons in lobsters: location, morphology, release of octopamine and possible physiological role. Cold Spring Harb Symp Ouant Biol 40:127-133.

Lefkowitz RJ (1998) G protein-coupled receptors. III. New roles for receptor kinases and beta-arrestins in receptor signaling and desensitization. J Biol Chem 273:18677-18680.

Lisman J (1994) The CaM kinase II hypothesis for the storage of synaptic memory. Trends Neurosci 17:406-412.

Livingstone M, Harris-Warrick R, Kravitz E (1980) Serotonin and octopamine produce opposite postures in lobsters. Science 208:76-79.

Mauelshagen J, Parker GR, Carew TJ (1996) Dynamics of induction and expression of long-term synaptic facilitation in Aplysia. J Neurosci 16:7099-7108.

Mauelshagen J, Sherff CM, Carew TJ (1998) Differential induction of long-term synaptic facilitation by spaced and massed applications of serotonin at sensory neuron synapses of Aplysia californica. Learn Mem $5: 246-256$

Muller U, Carew TJ (1998) Serotonin induces temporally and mechanistically distinct phases of persistent PKA activity in Aplysia sensory neurons. Neuron 21:1423-1434.

Mulloney B, Hall WM (1990) GABA-ergic neurons in the crayfish nervous system: an immunocytochemical census of the segmental ganglia and stomatogastric system. J Comp Neurol 291:383-394.

Mulloney B, Acevedo LD, Bradbury AG (1987) Modulation of the crayfish swimmeret rhythm by octopamine and the neuropeptide proctolin. J Neurophysiol 58:584-597.

Nishizuka Y (1995) Protein kinase C and lipid signaling for sustained cellular responses. FASEB J 9:484-496.

Olson GC, Krasne FB (1981) The crayfish lateral giants as command neurons for escape behavior. Brain Res 214:89-100.

Schneider H, Trimmer BA, Rapus J, Eckert M, Valentine DE, Kravitz EA (1993) Mapping of octopamine-immunoreactive neurons in the central nervous system of the lobster. J Comp Neurol 329:129-142.

Soderling TR (1999) The Ca-calmodulin-dependent protein kinase cascade. Trends Biochem Sci 24:232-236.

Stamford JA, Davidson C, McLaughlin DP, Hopwood SE (2000) Control of dorsal raphe 5-HT function by multiple 5- $\mathrm{HT}_{1}$ autoreceptors: parallel purposes or pointless plurality? Trends Neurosci 23:459-465.

Vu ET, Krasne FB (1993) Crayfish tonic inhibition: prolonged modulation of behavioral excitability by classical GABAergic inhibition. J Neurosci 13:4394-4402.

Wayman GA, Tokumitsu H, Soderling TR (1997) Inhibitory cross-talk by cAMP kinase on the calmodulin-dependent protein kinase cascade. J Biol Chem 272:16073-16076.

Weng G, Bhalla US, Iyengar R (1999) Complexity in biological signaling systems. Science 284:92-96.

Yau KW (1994) Cyclic nucleotide-gated channels: an expanding new family of ion channels. Proc Natl Acad Sci USA 91:3481-3483.

Yeh SR, Fricke RA, Edwards DH (1996) The effect of social experience on serotonergic modulation of the escape circuit of crayfish. Science 271:366-369

Yeh SR, Musolf BE, Edwards DH (1997) Neuronal adaptations to changes in the social dominance status of crayfish. J Neurosci 17:697708 . 